\title{
Value and limitations of adenosine in the diagnosis and treatment of narrow and broad complex
} tachycardias

\author{
A C RANKIN, K G OLDROYD, E CHONG, A P RAE, S M COBBE \\ From the University Department of Medical Cardiology, Royal Infirmary, Glasgow
}

SUMMARY The diagnostic and therapeutic potential of intravenous adenosine was studied in 64 patients during 92 episodes of regular sustained tachycardia. In 40 patients who had narrow complex tachycardias (QRS $<0.12 \mathrm{~s})$ adenosine $(2.5-25 \mathrm{mg}$ ) restored sinus rhythm in 25 with junctional tachycardias (46 of 48 episodes) and produced atrioventricular block to reveal atrial or sinus tachycardia in 15 . In 24 patients with broad complex tachycardias (QRS $\geqslant 0.12 \mathrm{~s}$ ) adenosine terminated the tachycardias in six patients and revealed atrial or sinus arrhythmias in four. The tachycardias persisted in 14 patients despite doses up to $20 \mathrm{mg}$, but adenosine allowed the diagnosis of ventricular tachycardia with retrograde atrial activation in two patients by producing transient ventriculoatrial dissociation. Diagnosis based on adenosine induced atrioventricular nodal block was correct in all patients with narrow complex tachycardias and in $92 \%$ of those with broad complex tachycardias, compared with correct electrocardiographic diagnoses in $90 \%$ and $75 \%$ respectively. Adenosine gave diagnostic information additional to the electrocardiogram in $25 \%$. The response to adenosine in broad complex tachycardias identified those of supraventricular origin with $90 \%$ sensitivity, $93 \%$ specificity, and $92 \%$ predictive accuracy. Adenosine restored sinus rhythm in all patients with junctional reentrant tachycardias, but in $10(35 \%)$ the arrhythmias recurred within two minutes. Symptomatic side effects (dyspnoea, chest pain, flushing, headache) were reported by $40(63 \%)$ patients and, although transient, were severe in 23 $(36 \%)$. There were ventricular pauses of over $2 \mathrm{~s}$ in $16 \%$ of patients, the longest pause being $6 \cdot 1 \mathrm{~s}$.

Adenosine is of value in the diagnosis and treatment of narrow and broad complex tachycardias, but its use is limited by symptomatic side effects, a tenfold range in minimal effective dosage, occasional action at sites other than the atrioventricular node, and early recurrence of arrhythmia.

Adenosine is a naturally occurring, rapidly metabolised compound that produces transient atrioventricular nodal block in humans when injected intravenously. ${ }^{1}$ It can terminate reentrant supraventricular tachycardias that involve the atrioventricular node, ${ }^{2-}$ while in tachycardias of atrial origin it may be of diagnostic value, because adenosine induced atrioventricular block slows the ventricular rate and reveals the unaffected atrial arrhythmia. ${ }^{2}$ Such diagnostic and therapeutic effects of adenosine should be of most value in broad complex

Requests for reprints to Dr A C Rankin, Department of Medical Cardiology, Royal Infirmary, 10 Alexandra Parade, Glasgow G31 2ER.

Accepted for publication 11 April 1989 tachycardias (which are often misdiagnosed ${ }^{5}$ ) by acting on supraventricular tachycardias with aberrant conduction, while having no effect on ventricular tachycardia. ${ }^{6}$ The brief duration of action of adenosine is of particular advantage in this context, making it a safer alternative to verapamil. ${ }^{7}$ The diagnostic use of adenosine based on its property of blocking the atrioventricular node may be questioned, however, because adenyl compounds can also act on accessory pathways, ${ }^{89}$ and may terminate some atrial ${ }^{10}$ and ventricular ${ }^{11}$ tachycardias. We therefore studied the clinical value of adenosine in patients with regular narrow or broad complex tachycardias to determine whether it provides diagnostic information additional to the surface electrocardiogram and to assess its therapeutic value. 


\section{Patients and methods}

Adenosine was administered to 64 patients ( 36 men and 28 women, aged 16-79 years (mean 51 years)), during 92 episodes of sustained regular tachycardia. Fifty four patients were studied during spontaneous episodes of tachycardia, on 75 occasions, while in 15 patients the tachycardias were induced by programmed stimulation; five patients received adenosine during both spontaneous and induced tachycardias. Electrocardiographic diagnoses were based on standard criteria for narrow ${ }^{1213}$ and broad ${ }^{14}$ complex tachycardias. Electrophysiological studies, with standard protocols, ${ }^{15}$ confirmed the diagnoses in 27 patients.

An adenosine (Sigma) solution $(5 \mathrm{mg} / \mathrm{ml}$ ) was prepared in sterile saline. Boluses of $2.5-25 \mathrm{mg}$ were administered by rapid intravenous injection via arm veins in most patients or into femoral or central veins in 16 patients. The dose was increased, to a maximum of $20 \mathrm{mg}$, by 2.5 or $5 \mathrm{mg}$ after the effects of the preceding dose had passed, until there was an effect on the arrhythmia or the patient reported intolerable side effects. One patient with recurrent supraventricular tachycardia required an additional $25 \mathrm{mg}$ bolus to terminate the arrhythmia. A continuous electrocardiogram was recorded during and for a minute after the administration of each dose. Blood pressure was measured by cuff sphygmomanometer or by intra-aortic pressure recording in patients undergoing electrophysiological study. Side effects were assessed by direct questioning and were scored by the patient from 0 to 10 ("very mild" to "very severe").
When the diagnostic value of adenosine was assessed it was assumed that it had its main action on the atrioventricular node and thus $(a)$ reentrant junc- $\bar{c}$ tional tachycardias would be terminated, $(b)$ atrial $\frac{}{\sigma}$ and sinus tachycardia would persist and be exposed $\frac{\bar{\sigma}}{\vec{\sigma}}$ when the ventricular rate was slowed by atrio- $\stackrel{\mathbb{Q}}{\Omega}$ ventricular block, and $(c)$ ventricular tachycardia would be unaffected. The mode of termination of junctional tachycardias was examined to determine the site of action of adenosine within the reentrant $\vec{\omega}$ circuit. The post-tachycardia sinus beats were $\stackrel{\mathscr{S}}{ }$ examined for pre-excitation, because conduction down an accessory pathway may be enhanced by adenosine induced atrioventricular block. The diag- $\omega$ nostic yield from the responses to adenosine was $\vec{\varphi}$ compared with the electrocardiographic diagnoses $o r$ and the results of electrophysiological study.

Results were expressed as means and standard deviations and the $t$ test was used to test for differences between means.

\section{Results}

\section{NARROW COMPLEX TACHYCARDIAS}

Forty patients with narrow complex tachycardias received adenosine during 64 episodes -55 spontaneous and nine induced at electrophysiological study. The QRS duration ranged from 0.04 to $0.08 \mathrm{~s}$ (mean $0.07 \mathrm{~s}$ ) and the tachycardia rates from 120 to 240 beats $/ \mathrm{min}$ (mean 170 beats $/ \mathrm{min}$ ). Adenosine produced an effect in all patients with narrow complex tachycardias, restoring sinus rhythm in 25 patients (46 of 48 episodes) (fig 1 ) and producing atrioventricular block to reveal atrial or sinus

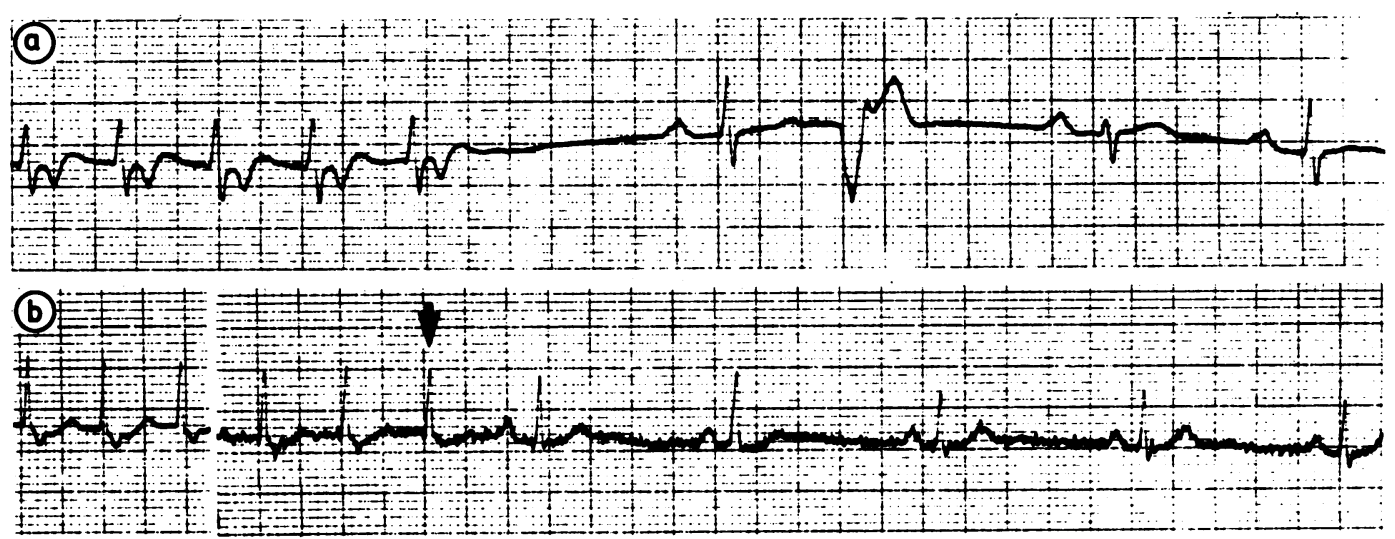

Fig 1 Termination of junctional tachycardias by adenosine. (a) In a patient with a concealed accessory pathway the tachycardia terminated 20 seconds after administration of $20 \mathrm{mg}$ adenosine. Adenosine blocked the anterograde limb of the reentrant circuit as retrograde atrial activation followed the last $Q R S$ complex of the tachycardia.

(b) Termination of junctional tachycardia by $20 \mathrm{mg}$ adenosine in a patient with dual atrioventricular nodal pathways.

The retrograde atrial activation seen during the tachycardia is absent after the last QRS complex of the tachycardia (arrowed) indicating block of the retrograde limb of the reentrant circuit. The initial three complexes are from tracings before adenosine. Somatic tremor artefact associated with adenosine induced symptoms. Paper speed $25 \mathrm{~mm} / \mathrm{s}$. 


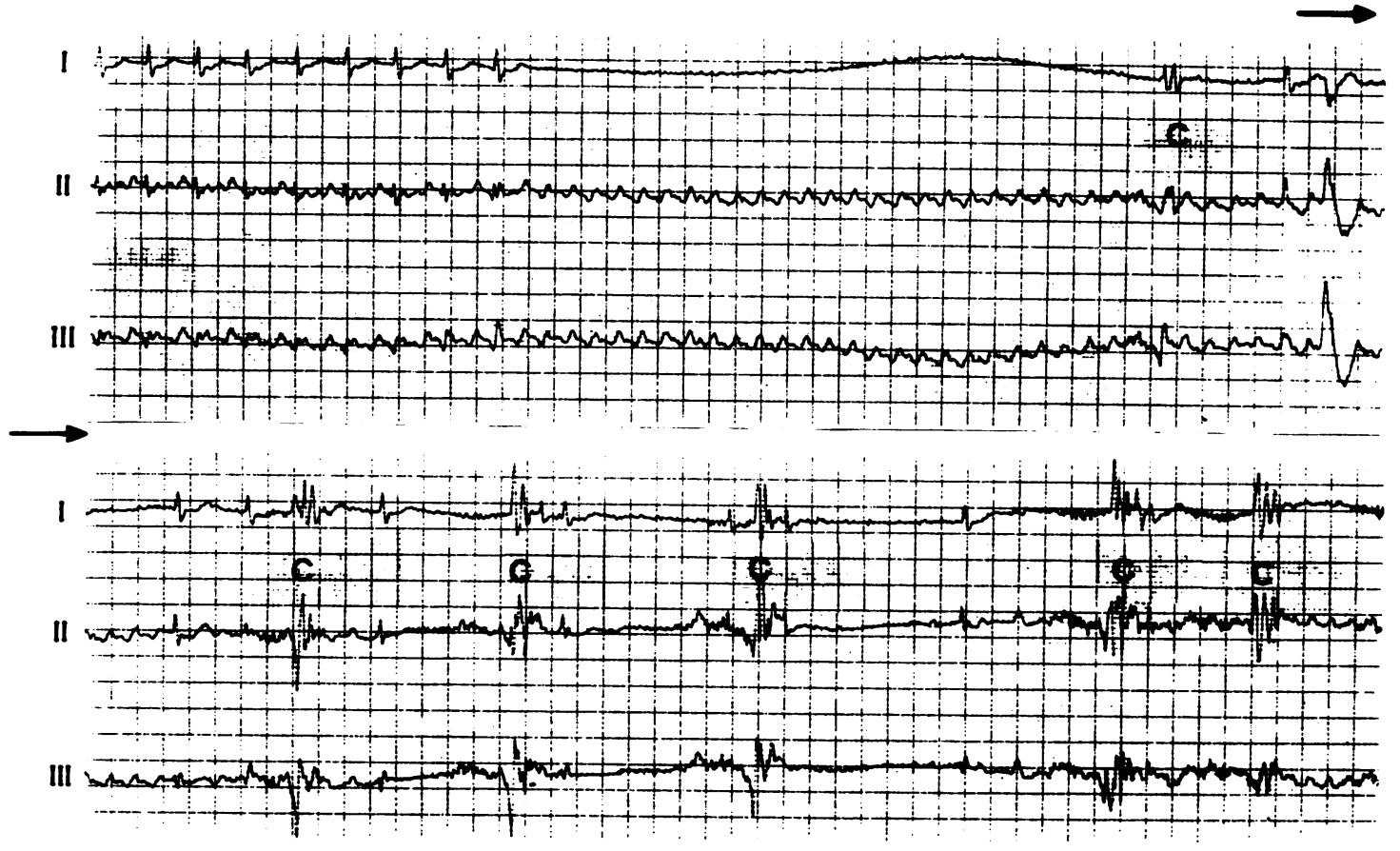

Fig 2 Continuous tracings in leads I, II, and III showing atrioventricular block and transient termination of atrial flutter by adenosine. Five seconds after adenosine administration atrioventricular block revealed flutter waves and resulted in ventricular standstill for $6 \cdot 1 \mathrm{~s}$. There were artefacts caused by coughs (C). Thirteen seconds after adenosine administration atrial flutter was terminated (bottom panel) but atrial flutter/fibrillation recurred. Paper speed $25 \mathrm{~mm} / \mathrm{s}$.

tachycardias in 15 patients (fig 2). The time to onset of the action of adenosine ranged from 5 to $40 \mathrm{~s}$ after intravenous injection (mean (SD) $19.5(8.3) \mathrm{s}$ ). The duration of effect in patients with atrial tachycardias was dose dependent, with atrioventricular block persisting for 6-30 s (mean $13 \mathrm{~s}$ ). There was a tenfold range in the minimum effective dose of adenosine, from 2.5 to $25 \mathrm{mg}$, with a mean (SD) dose of $8.8(6.2)$ mg. The dose varied between individuals but also depended on the route of administration. The mean (SD) dose to terminate the tachycardias was 3.0 (1.1) $\mathbf{m g}(\mathbf{n}=10)$ when administered via the femoral vein and $10.8(6.3) \mathrm{mg}(n=36)$ via peripheral veins $(p<$ 0.001).

An electrocardiographic diagnosis of probable junctional tachycardia was made in 24 patients with narrow complex tachycardias. Adenosine terminated the tachycardias in 22 of these patients, but revealed atrial tachycardia with first degree atrioventricular block in one and atrial flutter with $1: 1$ conduction in another. Five patients had tachycardias with $P$ waves preceding each QRS complex. Adenosine induced atrioventricular block confirmed sinus or atrial tachycardia in two with superior to inferior $P$ wave axes and terminated the tachycardia in the remaining three. Atrial flutter with 2:1 block was diagnosed from the electrocardiogram in 11 patients, and in each of these adenosine slowed the ventricular rate to reveal atrial flutter waves. Adenosine briefly terminated atrial flutter in one patient (fig 2).

Termination of junctional tachycardias by adenosine was the result of a block of the atrioventricular node in most patients, including 11 with accessory pathways (fig 1a). In four patients adenosine blocked the retrograde limb of the reentrant circuit, the last activity of the tachycardia being ventricular (fig $1 b$ ). This need not necessarily indicate an atrioventricular nodal tachycardia, as was shown by the patient in fig 3. Adenosine caused prolongation of the $\mathbf{A H}$ interval but the tachycardia was terminated by a block of retrograde conduction in what proved to be a concealed paraseptal accessory pathway of the less common slowly conducting type. An underlying diagnosis of Wolff-Parkinson-White syndrome was revealed by the response to adenosine in six patients, because the transient atrioventricular block enhanced pre-excitation owing to conduction down an accessory pathway (fig 4).

\section{BROAD COMPLEX TACHYCARDIAS}

Twenty four patients with broad complex tachycardias received adenosine during 28 episodes -20 


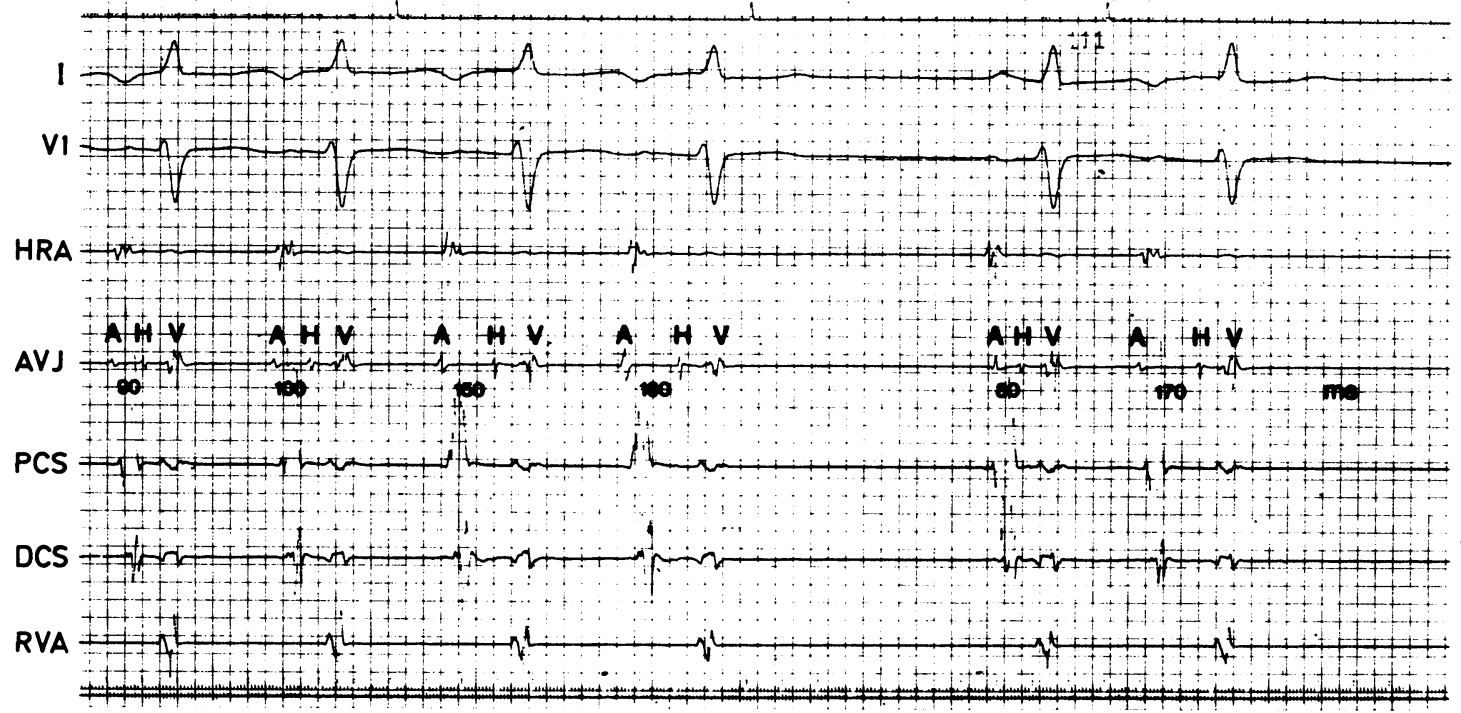

Fig 3 Termination of long RP junctional tachycardia by adenosine induced retrograde block. Twenty seconds after $2.5 \mathrm{mg}$ adenosine administration there was prolongation of the AH interval, but block then occurred retrogradely, after ventricular activation, as adenosine blocked conduction in a slowly conducting concealed paraseptal accessory pathway. After the post-tachycardia pause, both atrioventricular nodal and accessory pathway function recovered for the first sinus beat but the persisting adenosine effect produced AH prolongation and accessory pathway block with the subsequent reentrant beat. Surface leads I and VI. Intracardiac electrograms from high right atrium (HRA), atrioventricular junction (AVJ), proximal coronary sinus (PCS), distal coronary sinus (DCS), and right ventricular apex (RVA). Paper speed $100 \mathrm{~mm} / \mathrm{s}$.

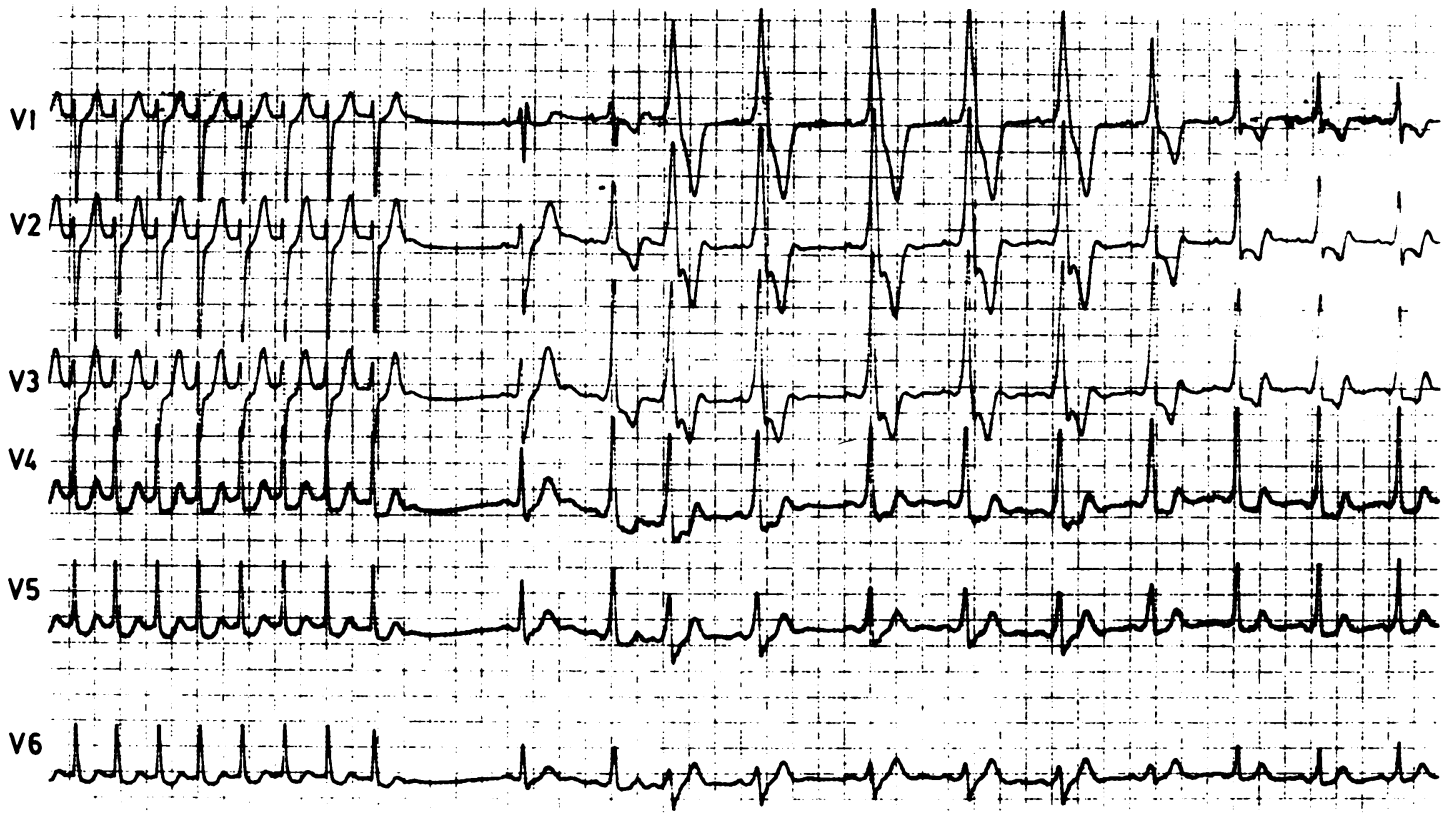

Fig 4 Pre-excitation after termination of junctional tachycardia by adenosine (2.5 mg) as transient atrioventricular block produced relative enhancement of conduction down a left sided accessory pathway. The first sinus beat was not overtly pre-excited, which may indicate transient block of accessory pathway conduction or may reflect delay in the onset of progressive atrioventricular nodal block. Chest leads V1-V6. Paper speed $25 \mathrm{~mm} / \mathrm{s}$. 
Time after adenosine $(20 \mathrm{mg})$
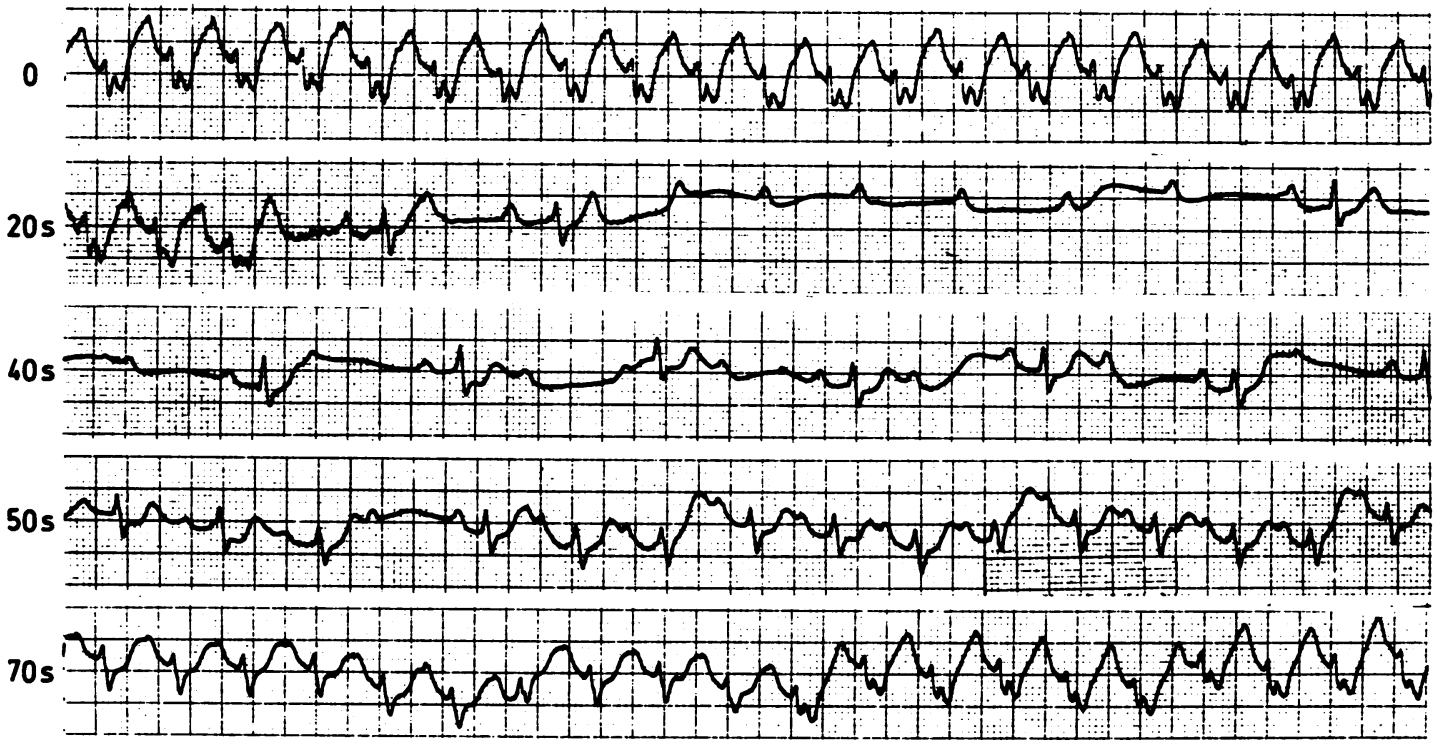

Fig 5 Broad complex tachycardia revealed as sinus tachycardia by adenosine. An arrhythmia with a configuration that was suggestive of ventricular tachycardia was terminated by the onset of atrioventricular block, with $5 \mathrm{~s}$ ventricular standstill, $20 \mathrm{~s}$ after administration of $20 \mathrm{mg}$ adenosine. The atrial rate then increased and atrioventricular conduction returned as the adenosine effect passed, with second degree block by $40 \mathrm{~s}$, first degree by $50 \mathrm{~s}$, and sinus tachycardia by $60 \mathrm{~s}$. At 70 s the rate was the same as before adenosine, but the complexes were narrow, until they became aberrant again at $80 \mathrm{~s}$ (middle lower panel). Lead II, paper speed $25 \mathrm{~mm} / \mathrm{s}$.

spontaneous and eight induced. The mean (SD) QRS duration was $0.14(0.03) \mathrm{s}$ and the tachycardia rates ranged from 110 to 240 beats/min (mean 162 beats $/ \mathrm{min}$ ). Adenosine terminated the tachycardias in six patients (eight of nine episodes) and induced atrioventricular block to reveal atrial or sinus tachycardias in four patients. As with narrow complex tachycardias, there was a wide range of minimum effective dosage, from 2.5 to $20 \mathrm{mg}$ (mean (SD) $11.0(5.6) \mathrm{mg})$. The tachycardias persisted despite doses of 10 to $20 \mathrm{mg}$ intravenous adenosine (mean (SD) $17.7(4.2) \mathrm{mg}$ ) in the remaining 14 patients.

The patients with broad complex tachycardia were divided into two groups according to the relation between atrial and ventricular activity on the electrocardiogram. Atrioventricular dissociation was not apparent in 13 patients. Adenosine restored sinus rhythm in five patients in whom the electrocardiogram indicated junctional tachycardia with aberrant conduction and revealed unsuspected atrial flutter in two patients and sinus or atrial tachycardia in two (fig 5). Tachycardia persisted despite the administration of $20 \mathrm{mg}$ of adenosine in four patients, suggesting the diagnosis of ventricular tachycardia. This was confirmed in two patients, who had atrial activity after each QRS complex, by adenosine induced block of retrograde conduction and the production of ventriculoatrial dissociation (fig 6). Evidence of atrioventricular dissociation was present in the remaining 11 patients, suggesting ventricular tachycardia. Adenosine had no effect on the tachycardia in 10 of these patients, but in one the tachycardia briefly accelerated and then terminated in the first minute after adenosine administration. After the reinduction of the tachycardia, repeat administration of adenosine up to $20 \mathrm{mg}$ failed to terminate the arrhythmia.

\section{CLINICAL VALUE AND LIMITATIONS OF ADENOSINE}

The response to adenosine gave the correct basic diagnosis in all patients with narrow complex tachycardia and in $92 \%$ of those with broad complex tachycardia (table), while the electrocardiographic diagnosis was correct in $90 \%$ of narrow complex tachycardias and $75 \%$ of broad complex tachycardias. Adenosine gave diagnostic information additional to the electrocardiogram in 16 patients $(25 \%)$. In patients with broad complex tachycardias, adenosine produced atrioventricular block and terminated or revealed tachycardias of supraventricular origin, with a sensitivity of $90 \%$, specificity of $93 \%$, and predictive accuracy of $92 \%$. 

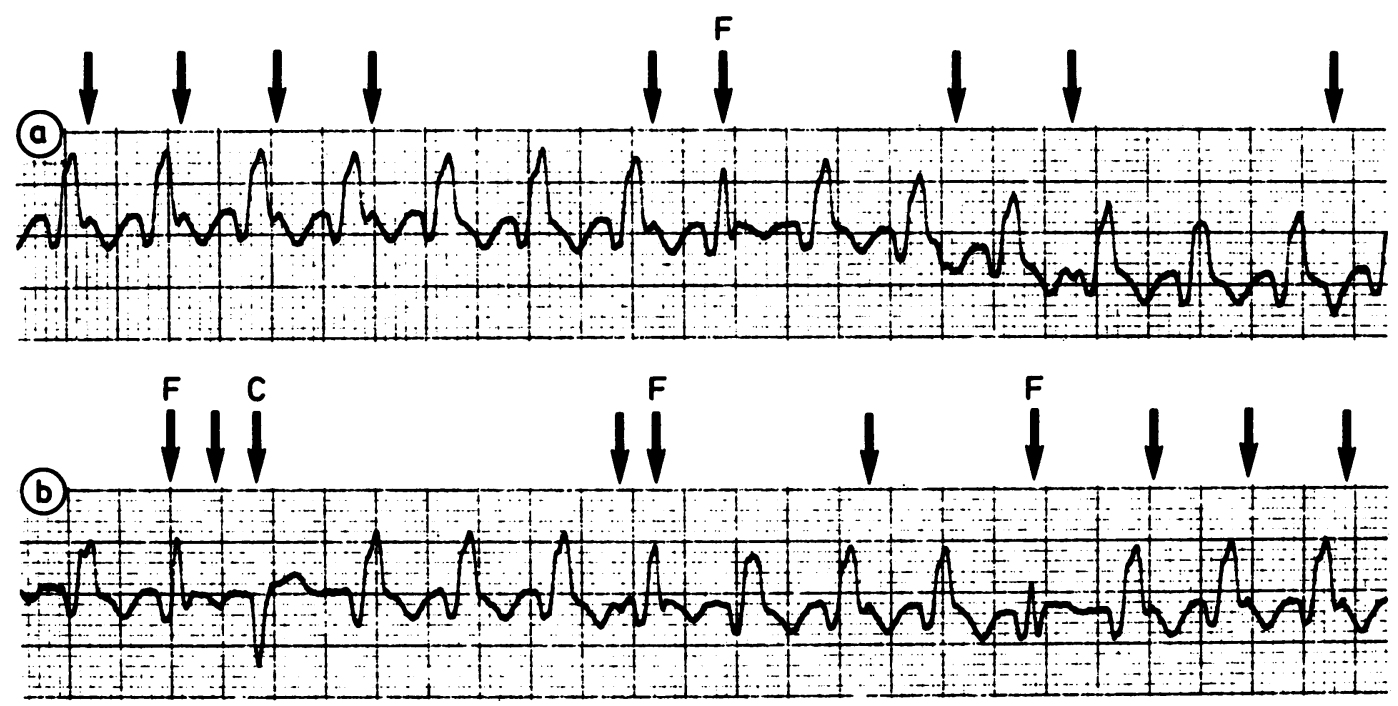

Fig 6 Adenosine induced ventriculoatrial block in a patient with ventricular tachycardia and 1:1 retrograde conduction (a) 30 s and (b) 45 s after adenosine (10 mg). The response to adenosine showed that the small notch following the first four $Q R S$ complexes was caused by atrial activity (unlabelled arrows), because it disappeared with the onset of atrioventricular block and the loss of retrograde ventriculoatrial conduction. The resultant dissociated atrial activity produced fusion beats $(F)$ (with narrowed $Q R S$ complexes) and a capture beat $(C)$ (with a configuration identical with that seen on return to sinus rhythm) before the recurrence of retrograde conduction (last three complexes). Lead V1, paper speed $25 \mathrm{~mm} / \mathrm{s}$.

Adenosine effectively restored sinus rhythm in all 29 patients with junctional reentrant tachycardias. However, the arrhythmia recurred within two minutes (mean $35 \mathrm{~s}$ ) of the restoration of sinus rhythm in $10(35 \%)$ of these patients (19 of 26 episodes). Electrocardiograms, obtained in 12 episodes, showed re-initiation of tachycardia by atrial extrasystoles in four patients, by ventricular extrasystoles in two, and by retrograde activation of the atria without preceding extrasystoles in three patients (fig 7). Five patients had sinus tachycardias (100 to 130 beats/ min) when their tachycardias were re-initiated by atrial or ventricular extrasystoles at a mean interval of $50 \mathrm{~s}$ after termination. By contrast, in four episodes when the arrhythmias were re-initiated by retrograde atrial activation without preceding extrasystoles, recurrence was earlier, at 6 to $15 \mathrm{~s}$ (mean $9 \mathrm{~s}$ ), and the mean heart rate was 46 beats/minute.
The negative chronotropic and dromotropic actions of adenosine may result in pronounced bradycardia if an excessive dose is administered (figs 2 and 5), but this did not occur in most patients. After termination of junctional tachycardias by adenosine, short pauses of up to $2.46 \mathrm{~s}$ occurred (mean ventricular pause $1.28 \mathrm{~s}$ ). More pronounced bradycardias were seen in patients with atrial or sinus arrhythmias in whom larger doses of adenosine induced atrioventricular block with ventricular pauses of up to $6.1 \mathrm{~s}$ (mean pause $1.9 \mathrm{~s}$ ). Ventricular pauses of more than $2 \mathrm{~s}$ occurred in $10(16 \%)$ patients. The recovery of heart rate was always rapid and the bradycardia was usually followed by sinus tachycardia.

Ventricular extrasystoles occurred in response to adenosine in $20(44 \%)$ of the 46 patients with supraventricular arrhythmias. Single ventricular

Table Diagnostic value of the electrocardiogram and responses to adenosine

\begin{tabular}{lllll}
\hline Arrhythmias & Junctional tachycardia & Atrial fiutter & Atrial or sinus tachycardia & Ventricular tachycardia \\
\hline Narrow complex: & $23 / 25$ & $11 / 12$ & $2 / 3$ & - \\
ECG & $25 / 25$ & $12 / 12$ & $3 / 3$ & - \\
Adenosine & & & & \\
Broad complex: & $4 / 5$ & $0 / 2$ & $2 / 3$ & $12 / 14$ \\
ECG & $5 / 5$ & $2 / 2$ & $2 / 3$ & $13 / 14$ \\
Adenosine & & & \\
\hline
\end{tabular}

Figures show the number of patients correctly diagnosed and the total number of patients with the diagnosis. See text for diagnostic criteria. 

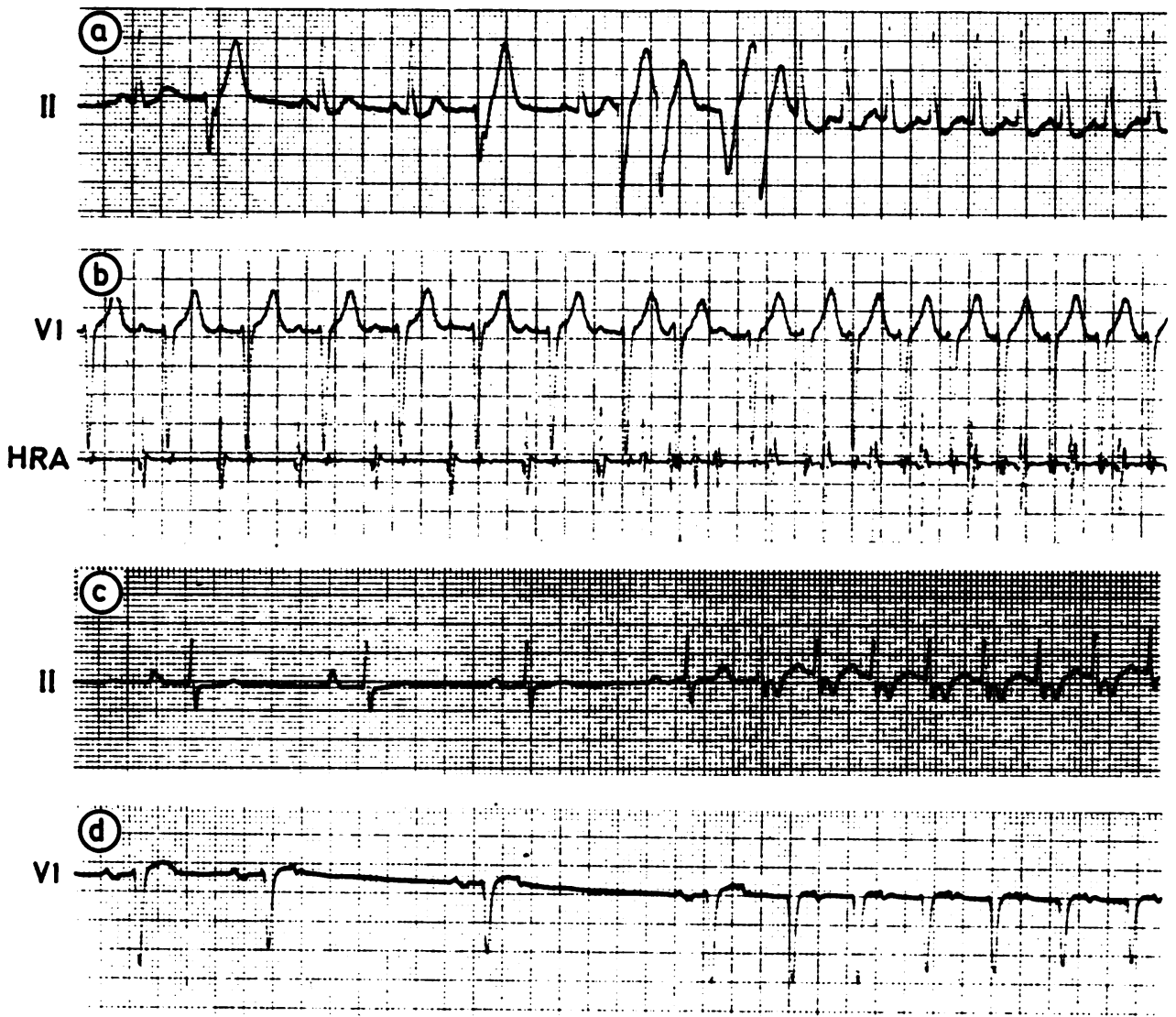

Fig 7 Early recurrence of junctional tachycardia after termination by adenosine. (a) Twenty seconds after restoration of sinus rhythm by $7.5 \mathrm{mg}$ adenosine a junctional tachycardia was re-initiated by ventricular extrasystoles. (b) Re-initiation of junctional tachycardia by multiple atrial extrasystoles in a patient with sinus tachycardia 30 s after restoration of sinus rhythm by $5 \mathrm{mg}$ adenosine. (c) Forty seconds after termination of junctional tachycardia by adenosine $7.5 \mathrm{mg}$ a single atrial extrasystole caused a further increase in $P R$ interval and re-initiated the tachycardia in a patient with resting sinus bradycardia and first degree heart block. (d) Ten seconds after restoration of sinus rhythm by $5 \mathrm{mg}$ adenosine retrograde atrial activation was seen after the sinus beats, and the third such beat re-initiated the tachycardia. Note the identical configuration of retrograde atrial beats before and after reinduction of tachycardia. Leads II or V1. HRA, high right atrial electrogram. Paper speed $25 \mathrm{~mm} / \mathrm{s}$.

extrasystoles occurred in nine patients, ventricular couplets in seven, and short episodes of non-sustained ventricular tachycardia in four. No persisting adverse effect resulted from these arrhythmogenic effects of adenosine, other than the involvement of ventricular extrasystoles in the re-initiation of junctional tachycardia in two patients (fig 7).

Symptomatic side effects were reported after adenosine $=$ was administered to $40(64 \%)$ of the patients. These included dyspnoea (36\%), chest pain ( $31 \%)$, flushing $(21 \%)$, and headache $(12 \%)$. Symp- toms were of similar short duration to the cardiac effects and had passed by one minute. Twenty three patients, however, described symptoms of more than moderate severity (scores of over 5/10), and 11 of them felt that the side effects were severe (scores of 8-10/10). Eighteen (29\%) reported multiple symptoms. No adverse haemodynamic response was seen in any of the patients including the six patients being treated with $\beta$ blockers. There was no change in the measured blood pressure other than increases after restoration of sinus rhythm. 
Discussion

The negative dromotropic action of intravenous adenosine in humans was first described in 1930 by Honey et al, but they concluded that they did "not regard adenosine as a useful therapeutic preparation for the treatment of heart disease" ${ }^{16}$ But the present results confirm more recent conclusions that adenosine is useful. ${ }^{2-4}$ The diagnostic and therapeutic effects of adenosine are attributable to its negative dromotropic action, mediated by a direct action on specific purine receptors in the heart. ${ }^{17}$ Its actions are not antagonised by atropine, but are blocked by aminophylline, ${ }^{1}$ and are probably caused by receptor mediated increases in potassium conductance, ${ }^{1819}$ with hyperpolarisation of the nodal cells. ${ }^{20}$ Side effects may also be the result of stimulation of specific purine receptors, with chest pain mediated by adenosine sensitive pain receptors, ${ }^{21}$ and stimulation of respiration secondary to an action on the carotid body. ${ }^{22}$ The rapid metabolism of adenosine in blood, with a half life in the circulation of about $10 \mathrm{~s}^{23}$ limits its use to the short term management of paroxysmal arrhythmias.

Although expert interpretation of the electrocardiogram will provide the correct diagnosis in most patients with tachycardias, ${ }^{13-15}$ it is recognised that such expertise is often not available. Many tachycardias, particularly those with broad complexes, are misdiagnosed. ${ }^{5}$ This may have serious therapeutic implications, because verapamil has been commonly administered to patients with ventricular tachycardia misdiagnosed as supraventricular with aberration. ${ }^{7}$ While the response to verapamil may also be of diagnostic value, ${ }^{24}$ the high incidence of adverse effects makes its use inadvisable. ${ }^{725}{ }^{26} \mathrm{By}$ contrast, the use of adenosine in the acute diagnosis and treatment of broad complex tachycardias seems to be safe. Adenosine has been reported to have a similar diagnostic value in regular broad complex tachycardias initiated by programmed stimulation, when the diagnosis of ventricular tachycardia is based solely on the lack of an effect of adenosine. ${ }^{6}$ The present results additionally show that adenosine may allow a positive diagnosis of ventricular tachycardia in those patients with 1:1 retrograde atrial activation, by producing ventriculoatrial dissociation.

The responses to adenosine in patients with narrow complex tachycardias allowed correct diagnosis in the few in whom the electrocardiogram had been misleading, and also indicated a possible anatomical basis for junctional tachycardias by showing pre-excitation in sinus rhythm. Tachycardia may be terminated by retrograde block in atrioventricular nodal tachycardias, ${ }^{2}$ but it cannot be considered as diagnostic of a nodal basis to the arrhythmia because accessory pathways may also be blocked by adeno- $\stackrel{C}{\mathrm{C}}$ sine and other adenyl compounds. ${ }^{89}$ The termination $\overrightarrow{\vec{F}}$ of atrial flutter by adenosine might also be mislead- $\stackrel{\rho}{?}$ ing, particularly in the absence of prior atrioven- $\frac{\text { ? }}{2}$ tricular block, as may occur with conduction down an $\frac{\bar{\sigma}}{\bar{\omega}}$. accessory pathway. ${ }^{6}$ Similarly, transient termination $\frac{{ }^{\circ}}{\sigma}$ of atrial tachycardia by adenosine triphosphate has $\varrho$ been reported, ${ }^{10}$ emphasising that termination of $\%$ arrhythmia by adenyl compounds is not completely $\overrightarrow{0}$ specific for junctional tachycardia. The termination of ventricular tachycardia after adenosine in our $\vec{\omega}$ patient was probably not a direct action of adenosine, but secondary to the positive chronotropic influences? that commonly follow the negative effects of adeno- $N$ sine. ${ }^{27} 23$ Adenosine, however, has been shown to $\omega$ terminate reproducibly catecholamine or exercise $\vec{f}$ induced ventricular tachycardia that is thought to be $\circ$ due to triggered activity mediated by cyclic adenosine monophosphate in patients with normal $\vec{f}$ hearts. ${ }^{611}$ Thus termination of arrhythmia by adeno- $\frac{\mathbb{D}}{0}$ sine is neither completely diagnostic of junctional $\mathbb{\Phi}$ tachycardia, nor even of a tachycardia of supraventricular origin.

Adenosine restored sinus rhythm in all patients $\vec{\oplus}$ with junctional tachycardias, but arrhythmia quickly $\underset{0}{\infty}$ recurred in a third of them. This seems to be attributable to more than the short duration of action and lack of persisting antiarrhythmic effect. Adenosine induced bradycardia is followed by tachycardia, presumably mediated by the sympathetic nervous $\frac{\mathrm{Q}}{\mathrm{O}}$ system, possibly owing to carotid body stimulation $\stackrel{\varrho}{\rightleftharpoons}$ by adenosine,,$^{28}$ and catecholamine induced extra- ${ }_{3}$ systoles which may have triggered the tachycardias. Earlier recurrences, during the bradycardiac phase of adenosine, may have resulted from a critical degree of

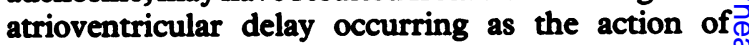
adenosine passed, the slowed anterograde conduc- $\frac{\varrho}{?}$ tion allowing recovery of the retrograde pathway and subsequent atrial activation. The high rate of recurrence may reflect the patient population, as some of these patients had troublesome recurrent arrhythmias, and such a high recurrence rate has not been윽 reported before. ${ }^{2-4}$ In two of these patients with $>$ recurrence after adenosine, however, the tachycardias could be terminated by atrial pacing without $\tilde{S}^{-}$ early recurrence of arrhythmia, suggesting that adenosine may have been provoking re-initiation.

Adenosine is effective in the diagnosis and treat $-\tilde{\omega}$ ment of both narrow and broad complex tachycardias. Its value is limited by occasional action at siteso other than the atrioventricular node and early recurrence of junctional tachycardia after restoration ${ }^{-}$ of sinus rhythm. The dose must be increased cautiously because of the high incidence of transient? but occasionally severe side effects and the wide? range of minimal effective dosage. An importanto 
clinical application of adenosine is in broad complex tachycardias in patients in whom the diagnosis is in doubt. Adenosine provides a safe means of diagnosis and treatment in such patients.

We thank our colleagues in the Department of Medical Cardiology for their cooperation in this study, the Pharmacy Department, University Hospital of Wales, Cardiff, for advice, and I T Calder, Pharmacist, Royal Infirmary, Glasgow, for preparing the sterile drug solutions.

\section{References}

1 Favale S, Di Biase M, Rizzo U, Belardinelli L, Rizzon P. Effect of adenosine and adenosine-5-triphosphate on atrioventricular conduction in patients. J Am Coll Cardiol 1985;5:1212-9.

2 Di Marco JP, Sellers TD, Lerman BB, Greenberg ML, Berne RM, Belardinelli L. Diagnostic and therapeutic use of adenosine in patients with supraventricular tachyarrhythmias. J Am Coll Cardiol 1985;6: 417-25.

3 Munoz A, Leenhart A, Sassine A, Galley P, Puech P. Therapeutic use of adenosine for terminating spontaneous paroxysmal supraventricular tachycardia. Eur Heart J 1984;5:735-8.

4 Watt AH, Bernard MS, Webster J, Passani SL, Stephens MR, Routledge PA. Intravenous adenosine in the treatment of supraventricular tachycardia: a dose-ranging study and interaction with dypyridamole. Br J Clin Pharmacol 1986;21:227-30.

5 Dancy M, Camm AJ, Ward D. Misdiagnosis of chronic recurrent ventricular tachycardia. Lancet 1986;il: 320-3.

6 Griffith MJ, Linker NJ, Ward DE, Camm AJ. Adenosine in the diagnosis of broad complex tachycardia. Lancet 1988;i:672-5.

7 Rankin AC, Rae AP, Cobbe SM. Misuse of intravenous verapamil in patients with ventricular tachycardia. Lancet 1987;i1:472-4.

8 Perrot B, Clozel JP, Faivre G. Effect of adenosine triphosphate on the accessory pathway. Eur Heart $J$ 1984;5:382-93.

9 Rinne C, Sharma AD, Klein GJ, Yee R, Szabo T. Comparative effects of adenosine triphosphate on accessory pathway and atrioventricular nodal conduction. Am Heart J 1988;115:1042-7.

10 Perelman MS, Krikler DM. Termination of focal atrial tachycardia by adenosine triphosphate. Br Heart J 1987;58:528-30.

11 Lerman BB, Belardinelli L, West A, Berne RM, DiMarco JP. Adenosine-sensitive ventricular tachycardia: evidence suggesting cyclic AMPmediated triggered activity. Circulation 1986;74: $270-80$.

12 Wu D, Denes P, Amat-Y-Leon F, et al. Clinical, electrocardiographic and electrophysiologic observations in patients with paroxysmal supraventricular tachycardia. Am J Cardiol 1978;41:1045-50.

13 Bär FW, Brugada P, Dassen WRM, Wellens HJJ. Differential diagnosis of tachycardia with narrow QRS complex (shorter than 0.12 second). Am J Cardiol 1984;54:555-60.

14 Wellens HJJ, Bär FWHM, Lie KI. The value of the electrocardiogram in the differential diagnosis of a tachycardia with a widened QRS complex. Am J Med 1978;64:27-33.

15 Josephson ME, Seides SF. Clinical cardiac electrophysiology. Techniques and interpretations. Philadelphia: Lea and Febiger, 1979:23-59.

16 Honey RM, Ritchie WT, Thomson WAR. The action of adenosine upon the humạn heart. $Q J$ Med 1930; 23:485-9.

17 Burnstock G. Purinergic receptors in the heart. Circ Res 1980;46(suppl I):175-82.

18 Belardinelli $\mathrm{L}$, Isenberg $\mathrm{G}$. Isolated atrial myocytes: adenosine and acetylcholine increase potassium conductance. Am J Physiol 1983;244:H734-7.

19 Hutter OF, Rankin AC. Ionic basis of the hyperpolarising action of adenyl compounds on sinus veriosus of the tortoise heart. J Physiol 1984;353:111-25.

20 West GA, Belardinelli L. Correlation of sinus slowing and hyperpolarization caused by adenosine in sinus node. Pflugers Arch 1985;403:75-81.

21 Sylvén C, Jonzon B, Brandt R, Beermann B. Adenosine-provoked angina pectoris-like pain-time characteristics, influence of autonomic blockade and naloxone. Eur Heart J 1987;8:738-43.

22 Watt AH, Reid PG, Stephens MR, Routledge PA. Adenosine-induced respiratory stimulation in man depends on site of infusion. Evidence for an action on the carotid body? $\mathrm{Br} J$ Clin Pharmacol 1987;23: 486-90.

23 Klabunde RE. Dipyridamole inhibition of adenosine metabolism in human blood. Eur J Pharmacol 1983; 93:21-6.

24 Morgan DE, Brennan FJ. Diagnostic potential for verapamil in wide QRS complex tachycardia. Am J Cardiol 1985;55:1428-9.

25 Stewart RB, Bardy GH, Greene HL. Wide complex tachycardia: misdiagnosis and outcome after emergent therapy. Ann Intern Med 1986;194:766-71.

26 Buxton AE, Marchlinski FE, Doherty JU, Flores B, Josephson ME. Hazards of intravenous verapamil for sustained ventricular tachycardia. Am J Cardiol 1987; 59:1107-10.

27 Di Marco JP, Sellers TD, Berne RM, West GA, Belardinelli L. Adenosine: electrophysiologic effects and therapeutic use for terminating paroxysmal supraventricular tachycardia. Circulation 1983;68: $1254-63$.

28 Watt AH, Routledge PA. Transient bradycardia and subsequent tachycardia produced by intravenous adenosine in healthy adult subjects. $\mathrm{Br} J \mathrm{Clin}$ Pharmacol 1986;21:533-6. 